\title{
Einleitung: „Philosophie der Stadt“
}

\section{Stadt und Urbanität als Herausforderungen der Praktischen Philosophie.}

\section{Introduction: "Philosophy of the City" \\ Practical Philosophy, Urbanity, and the City}

\section{Andrea Klonschinski, Göttingen \& Johannes Müller-Salo, Hannover}

Zusammenfassung: Obwohl sich die „Philosophie der Stadt“ in den letzten Jahrzehnten dynamisch entwickelt hat, stellt sie noch keine klar konturierte philosophische Teildisziplin dar. Dieser Zustand birgt Chancen und Probleme: Die aktuellen Debatten sind vielschichtig und kreativ, die Potentiale verschiedener Theorien, Traditionen und Methoden zur philosophischen Erschließung der Stadt werden erprobt und kombiniert. Zugleich zeigt sich nach wie vor eine gewisse Unsicherheit hinsichtlich der genauen Ziele und Aufgaben einer Philosophie der Stadt sowie hinsichtlich ihres Verhältnisses zu anderen Disziplinen der Stadtforschung. Die Einleitung in den Schwerpunkt (i) gibt Einblicke in den Stand der Debatte, (ii) arbeitet auf dieser Basis drei Ansätze der Beschreibung der Ziele und Aufgaben einer Philosophie der Stadt heraus, und (iii) ordnet die Beiträge des Schwerpunktes in diese Systematik ein.

Schlagwörter: Stadt, Philosophie der Stadt, Urbanismus, Gerechtigkeit, Partizipation

Abstract: Although the philosophy of the city has developed dynamically within the last decade, it cannot be considered a well-established philosophical sub-discipline yet. As such, it shares all the strengths and weaknesses of an evolving field: The debates are diverse and creative. Philosophers working in the field test the usefulness of a wide variety of theories, traditions, and methods to further the city's philosophical understanding and interpretation. At the same time, there still exists some uncertainty regarding the exact goals and tasks of a philosophy of the city and its location within the broad and interdisciplinary field of urban research and urban theory. The 
present introduction to the special issue (i) provides insights into the current state of the debate, (ii) elaborates three different but mutually compatible accounts of a philosophy of the city, and (iii) pigeonholes the contributions to this special issue within this framework.

Keywords: City, Philosophy of the City, Urbanism, Justice, Participation

Menschliches Leben ist immer häufiger städtisches Leben. Bereits jetzt lebt gut die Hälfte der Menschheit in Städten. Nach Prognosen der Vereinten Nationen werden in knapp zehn Jahren sechzig und in der Mitte des 21. Jahrhunderts 70 Prozent aller Menschen in Städten und urbanen Zentren leben. ${ }^{1}$ Während hierzulande gelegentlich die Vermutung geäußert wird, die Corona-Pandemie - vor allem aber die durch sie erzwungenen digitalen und damit weniger standortgebundenen Reorganisationen der Arbeitswelt - werde Prozesse der Landflucht und Verstädterung verlangsamen oder gar umkehren, kann am Fortbestehen eines weltweiten Trends der Urbanisierung vor allem mit Blick auf den globalen Süden kein Zweifel bestehen.

Die Zukunft der Menschen liegt also überwiegend in den Städten, deren nur teilweise geplantes, oftmals aber unkontrolliert und chaotisch verlaufendes Wachstum eine Fülle von Chancen birgt, aber auch Probleme und Konflikte produziert. Um das Ausmaß der Herausforderungen zu umreißen, seien hier exemplarisch drei Aspekte genannt, die für die gegenwärtige Entwicklung von Städten auf allen Kontinenten von zentraler Bedeutung sind: Erstens ist städtischer Raum knapper Raum, wodurch permanent neue Verteilungskonflikte entstehen. Diese können als ein wichtiger Bestandteil umfassenderer urbaner Auseinandersetzungen um Gerechtigkeit gedeutet werden: Wer darf welchen Raum zu welchen Bedingungen nutzen? Welchen Stellenwert besitzt der öffentliche Raum, der für alle kostenlos und im Wortsinn, d.h. ohne einschränkende physische Barrieren, zugänglich ist bzw. sein sollte? Über wie viel städtischen Raum darf eine Person als Privateigentum

1 United Nations, Department of Economic and Social Affairs, Population Division (2018), The World's Cities in 2018-Data Booklet, https://www.un.org/ en/events/citiesday/assets/pdf/the_worlds_cities_in_2018_data_booklet. pdf, Vorwort, abgerufen am 27.4.2021.

2 Für eine rechtswissenschaftliche Auseinandersetzung mit dem Recht auf öffentlichen Raum, die umfangreich stadttheoretische Forschungen und Fragestellungen miteinbezieht, siehe Siehr (2017); dazu auch Young (1990, 225-256). 
verfügen und welche Grenzen setzt die Sozialpflichtigkeit des Eigentums der freien Verfügbarkeit über diesen Raum mit Blick auf so unterschiedliche Fragen wie die Höhe der Mietpreise und die äußere, ästhetische Gestaltung? Die in sehr unterschiedlichen Kontexten geführte und sehr verschiedene Dimensionen umfassende Diskussion um Teilhabegerechtigkeit, Inklusion und Partizipation ist durch die genannten Aspekte eng mit der Auseinandersetzung um das „Recht auf Stadt“, wie Henri Lefebvre es prominent formulierte, also um das Recht auf Nutzung und Inbesitznahme des städtischen Raums, verknüpft. ${ }^{3}$

Zweitens sind es die Städte, in denen sich primär entscheiden wird, ob und wie schnell die Wende hin zu einer klimaneutralen Lebens- und Wirtschaftsweise gelingt und mit welchen sozialen Folgekosten sie verbunden ist (vgl. allgemein zum Thema Stadt und Klimakrise Calthorpe 2013; Dekker 2020; Fields und Renne 2021). Die Herausforderungen des Klimaschutzes reichen von einer umfassenden, sozial gerecht finanzierten, energetischen Sanierung der Bausubstanz über die Renaturierung städtischer Räume zur Stärkung der Widerstandsfähigkeit von Städten gegenüber Extremwetterereignissen bis hin zur völligen Umgestaltung städtischer Mobilität und städtischer Verkehrsformen, die mit einem profunden Wandel von Alltagskultur und alltäglichen städtischen Praktiken einhergeht. Bei der Bewältigung dieser Aufgaben dürfte digitalen Strategien im Rahmen sogenannter Smart-City-Konzepte eine entscheidende Bedeutung zukommen und es steht damit zu erwarten, dass sich das Leben in der Stadt ganz erheblich durch Digitalisierungsprozesse ändern wird. Zu den zentralen Aufgaben der kommenden Jahrzehnte wird es gehören, diese Wandlungsprozesse sozial gerecht auszugestalten. ${ }^{4}$

Drittens, die Städte sind nach wie vor der zentrale Ort nicht nur der Politik im institutionell-verfassten Sinn - Regierungen haben ihren Sitz in Städten -, sondern auch des Politischen im Sinne individueller und in Gruppen organisierter Formen der Teilhabe an politischen Aushandlungsprozessen, des Protestes und der zivilgesellschaftlichen Öffentlichkeit im Allge-

3 Klassisch Lefebvre (2016 [1968]). Das normative Konzept eines „Rechts auf Stadt" prägt die Stadtforschung seit einem halben Jahrhundert, vgl. exemplarisch Brenner (2011), Mitchell (2012), Harvey (2019), Horn (2020).

Vgl. exemplarisch die aus Fallstudien bestehende Untersuchung von McLaren und Agyeman (2017), die für ein Zusammendenken von urbanen Digitalisierungsprozessen mit Gerechtigkeitsfragen argumentieren. 
meinen. ${ }^{5}$ Als Orte kultureller und weltanschaulicher Vielfalt, der Erprobung neuer Lebensstile und der Bildung von Subkulturen und als Orte, in denen sich politische Bewegungen formieren und artikulieren, von der Arabellion über Pegida bis hin zu Fridays for Future und den selbst ernannten Querdenkern, spielen Städte eine wichtige Rolle für verschiedene gesellschaftliche Dynamiken. Es wird sich deswegen vor allem in Städten zeigen, ob und unter welchen Bedingungen ein friedliches und offenes Zusammenleben von Menschen äußerst verschiedener Herkunft, Religion, sozialer Zugehörigkeit, sexueller Identität und politischer Orientierung gelingt.

Angesichts dieser vielfältigen Herausforderungen kann es kaum überraschen, dass die Stadt philosophisches Interesse weckt. Überraschend scheint eher, dass so viel Zeit bis zur Herausbildung einer eigenen Subdisziplin „Philosophie der Stadt“ vergangen ist. Die damit implizit formulierte These, dass es sich bei der Philosophie der Stadt um ein neues, noch im Entstehen begriffenes Unterfangen handelt, bedarf sicher der Begründung. Denn natürlich hat sich die Philosophie in ihrer Geschichte immer wieder auch für die Stadt interessiert. Mehr noch, die Philosophie ist in gewisser Hinsicht selbst ein Kind der Stadt: Es waren die griechischen Kleinstaaten mit städtischem Kern, in denen die Philosophie entstand. Praktische Philosophie war zuallererst die Lehre von der Ethik, Ökonomik und Politik des Zusammenlebens in der Stadtgemeinschaft und nicht nur Sokrates suchte nach philosophischer Wahrheit auf den Straßen und Märkten der Stadt Athen. ${ }^{6}$

$5 \quad \mathrm{Zu}$ denken ist hier schon an Hannah Arendts Vita activa (2002), in der die Stadt in Gestalt einer idealisierten polis als genuiner Ort des Politischen, als Ort des gemeinsamen menschlichen Handelns bestimmt wird.

6 So antwortet Sokrates auf die Vorhaltung des Phaidros, dass er nur selten aus der Stadt hinaus ins Freie gehe: „Dies verzeih mir schon, o Bester. Denn ich bin eben lernbegierig, und Felder und Bäume wollen mich nichts lehren, wohl aber die Menschen in der Stadt." (Phaidros, 230d; Platon 1981, 15). Grundsätzlich muss bei jedem Anschluss moderner Stadtphilosophie an antike Vorstellungen, der nicht selten, wie etwa bei Arendt, stark idealisierende Züge trägt, mitbedacht werden, dass die altgriechische polis mit der modernen (Groß-)Stadt nur sehr begrenzt vergleichbar ist. $\mathrm{Zu}$ denken ist etwa an die Größenunterschiede, aber auch an den Umstand, dass zu den altgriechischen Kleinstadtstaaten in der Regel auch dörfliche, durch Agrarwirtschaft geprägte Landstriche gehören. 
Die Philosophie der Stadt ist insofern eine genuine Neuerscheinung, als sie sich vor allem für die Stadt selbst als Ort des Zusammenlebens interessiert und die Stadt nicht nur als Bühne für anderes, das philosophische Aufmerksamkeit verdient, sieht. Im frühen zwanzigsten Jahrhundert haben sich Autoren wie Georg Simmel und Walter Benjamin mit Prozessen urbaner Vergesellschaftung und städtischen Wandels beschäftigt (Simmel 2006; Benjamin 1982). Doch ihr vorrangiges Interesse galt der Moderne, der Entstehung und Veränderung moderner Gesellschaften. Die Stadt gewann ihre Aufmerksamkeit vor allem, da sie der Ort war, an dem Moderne stattfand. Von daher gibt es gute Gründe, die gegenwärtig unter dem Oberbegriff Philosophie der Stadt / philosophy of the city geführten Debatten als neu und nicht als Wiederaufnahme und Fortführung älterer philosophischer Diskurse zu interpretieren. Dies zeigt sich nicht zuletzt daran, dass „Klassiker“ der (Stadt-)Philosophie in diesen Debatten nur eine untergeordnete Rolle spielen, der systematische und umfassende Anschluss keinesfalls gesucht wird.

Wozu braucht es eine Philosophie der Stadt? Die Frage ist mehr als bloße Rhetorik, wenn man bedenkt, dass die Stadt seit längerer Zeit und aus unterschiedlichsten Perspektiven zum Gegenstand disziplinärer und interdisziplinärer Forschung gemacht wird. Neben den klassischerweise mit Phänomenen des Städtischen befassten Disziplinen wie der Stadtplanung und ihrer Theorie, den Verkehrsingenieurswissenschaften, der Stadtgeschichtsforschung, der Architektur sowie der Human- und Stadtgeographie existieren eine Soziologie der Stadt (vgl. etwa Eckardt 2004; Löw 2018), eine Anthropologie der Stadt (zum Einstieg Pardo 2012; Jaffe und De Koning 2016) sowie eine sich kulturwissenschaftlich verstehende interdisziplinäre Stadtforschung und eine mit dieser eng verknüpften urban theory. ${ }^{7}$ Der Chor ist bereits vielstimmig, die Notwendigkeit zusätzlicher, philosophischer Zwischentöne liegt nicht auf der Hand.

Ein Konsens darüber, wie die Frage nach dem Warum zu beantworten ist, ist bei denjenigen, die zur Philosophie der Stadt beitragen, nicht erkennbar. Vielmehr zeigt sich im Guten wie im Schlechten, dass die Phi-

$7 \quad$ Vgl. zu einer aktuellen Bestandsaufnahme Jayne und Ward (2017); für eine in der urban theory einflussreiche Fallstudie siehe Sassen (2001 [1991]). Wegweisende Arbeiten von Autor_innen wie Jane Jacobs (1961) und David Harvey (1973) gehören zu Grundlagentexten der urban theory. Für Ansätze zur Kanonbildung bzw. zur Bestimmung von Schlüsseltexten der Stadttheorie s. Eckardt (2017). 
losophie der Stadt als Forschungsfeld noch im Entstehen begriffen ist. ${ }^{8} \mathrm{Zu}$ den Vorzügen eines solchen frühen Diskussionsstandes zählen zweifelsohne Kreativität und Offenheit. Innerhalb der philosophischen Diskussion um die Stadt finden sich die unterschiedlichsten Theorieeinflüsse und theoretischen Collagen. Die Potentiale praktisch aller philosophisch einflussreichen Strömungen der Moderne - von der analytischen Philosophie über die Phänomenologie und den Existentialismus bis hin zum Marxismus, zur Kritischen Theorie, zur liberalen Gerechtigkeitstheorie und zur postmodernen französischen Philosophie -, zum besseren Verständnis des Phänomens Stadt beizutragen, werden erprobt. Dabei zeigt sich immer wieder die Bereitschaft, ungewöhnliche Theoriesynthesen zu erstellen. Selbstverständlich beeinflussen darüber hinaus auch zentrale sozial- und kulturwissenschaftliche Werke der Stadtforschung, von Jane Jacobs über Henri Lefebvre bis hin zu David Harvey, die Philosophie der Stadt.

Die Probleme der gegenwärtigen Diskussionslage hängen mit den genannten Vorzügen systematisch zusammen. Bisher ist nicht festzustellen, dass sich unter den Diskursteilnehmenden geteilte Überzeugungen hinsichtlich der Frage herausgebildet haben, welche Kategorien und Begriffe für die philosophische Auseinandersetzung mit Städten zentral und wie sie zu interpretieren sind. Vielmehr existiert ein Nebeneinander sehr unterschiedlicher Zugriffe, die bisweilen beliebig, zumindest aber schwankenden Moden und Trends unterworfen zu sein scheinen.

Die Philosophie der Stadt steht daher unserer Einschätzung nach vor der doppelten Aufgabe, ihren eigenen Forschungsgegenstand und damit ihr Verhältnis zu den anderen mit der Stadt befassten Disziplinen klar zu bestimmen und sich zugleich ihrer leitenden Begriffe und methodischen Zugriffe zu vergewissern. Die Fragen sind alles andere als einfach zu beantworten und natürlich werden sich, wie in der Philosophie üblich, deutlich voneinander abweichende, für sich genommen plausible Antworten entwickeln und verteidigen lassen.

Der Debattenstand ist also unübersichtlich. Das gilt ungeachtet der Tatsache, dass die Philosophie der Stadt seit ungefähr einem Jahrzehnt als eigenständiges Feld des Philosophierens immer sichtbarer geworden ist: Es

8 Vgl. zur Illustration der folgenden Bemerkungen die in den nächsten Absätzen genannten Heftschwerpunkte, Sammelbände und Kompendien, bei deren Lektüre deutlich wird, dass sich in der Philosophie der Stadt gegenwärtig die unterschiedlichsten Traditionen und Vorstellungen von Philosophie begegnen und wenig unversucht bleibt. 
gibt erste Kompendien zur Philosophie der Stadt, ${ }^{9}$ dem Thema sind Zeitschriftenschwerpunkte wie der vorliegende gewidmet, ${ }^{10}$ die wissenschaftliche Netzwerkbildung schreitet voran. ${ }^{11}$ Da sich aber, wie angedeutet, noch keine klar konturierte „Leitdebatte" entwickelt hat, von der ausgehend sich bestimmen ließe, welche zentralen Fragestellungen die Philosophie der Stadt gegenwärtig prägen, welchen Begriffen die Hauptaufmerksamkeit gilt und mit welchen Theorien und Traditionen gearbeitet wird, ist es nicht möglich, in dieser Schwerpunkteinleitung einen allgemeinen Überblick über den aktuellen Diskussionsstand zu geben. Wir wählen daher einen anderen Weg, indem wir den Versuch einer Systematisierung wagen: Nach unserer Einschätzung lassen sich aus den jüngeren Beiträgen drei verschiedene Auffassungen darüber, was die genuinen Aufgaben und Ziele einer Philosophie der Stadt sind, destillieren. Diese Dreiteilung wird aus der Beobachterperspektive vorgenommen: Wir behaupten damit also nicht, dass die von uns einer Auffassung zugeordneten Beiträge diese Auffassung selbst explizit verteidigen oder auch nur direkt thematisieren. Ebenso wenig gehen wir davon aus, dass sich die drei Auffassungen wechselseitig ausschließen und keine Überschneidungen zwischen ihnen möglich sind.

Einer ersten Auffassung zufolge ist unter „Philosophie der Stadt“ die Erweiterung und Anwendung etablierter Fragestellungen, Argumente und Methoden der Praktischen Philosophie auf den Gegenstandsbereich Stadt, Urbanisierung und städtisches Leben zu verstehen. Hier ließen sich zahlreiche Beispiele nennen. Ausgehend von allgemeinen Gerechtigkeitstheorien oder ethischen Überlegungen zur Inklusion lässt sich die Frage nach der Ausgestaltung einer gerechten Stadtgesellschaft stellen, die allen Bewohner_innen der Stadt angemessene Teilhabe ermöglicht und sie vor ungerechtfertigter Exklusion schützt. Unter Rückgriff auf politikphilosophische Konzeptionen von Repräsentation und Partizipation kann darüber nachge-

9 S. etwa das Textbuch von Meagher (2008) sowie die Bände von Jacobs und Malpas (2019) und von Meagher, Noll und Biehl (2019).

10 Vgl. aus den letzten Jahren etwa die von Hasse (2016), Epting (2019), Lehtinen $(2020 a ; 2020 b)$ und Simon (2021) herausgegebenen Zeitschriftenschwerpunkte.

11 Zu nennen ist hier vor allem Philosophy of the City Research Group (https:// philosophyofthecity.org/philosophy-of-the-city-research-group/), deren Konferenzen und Publikationen maßgeblich zur Entwicklung des Feldes beigetragen haben. 
dacht werden, wer zu welchem Zeitpunkt aus welchem Grund das Recht erhält, an lokalpolitischen Prozessen und Abstimmungen teilzunehmen, wann Entscheidungen durch repräsentative, gewählte Gremien und wann direktdemokratisch zu treffen sind. Eine Ästhetik wiederum, die sich für Umwelten, ihren Wert und die Standards ihrer Wahrnehmung interessiert, ${ }^{12}$ kann als Stadtästhetik nach dem Wert städtischer Umwelten und nach Prinzipien der Stadtgestaltung fragen. Arbeiten, die sich dieser ersten Lesart einer Philosophie der Stadt zuordnen lassen, stellen darauf ab, zentrale Aspekte städtischen Lebens und städtischer Praxis unter Rückgriff auf etablierte Theorien und Konzepte besser zu verstehen bzw. aufzuzeigen, auf Grundlage welcher normativen Überzeugungen und Regeln sie weiter zu gestalten und zu verbessern sind.

Nach einer zweiten Auffassung lässt sich die Philosophie der Stadt als Metadisziplin betrachten, die theoretischen wie begrifflichen Grundlagenfragen der einzelnen fachwissenschaftlichen wie auch interdisziplinär geführten Stadtdiskursen nachgeht. Philosophie der Stadt ist damit in erster Linie Wissenschaftsphilosophie der Stadtforschung. Eine so verstandene Philosophie der Stadt klärt zentrale Begriffe der Stadtforschung, wobei an erster Stelle natürlich der Begriff der Stadt selbst zu nennen ist: Lässt sich „Stadt" allgemein und zugleich informativ definieren, lassen sich hinreichende und notwendige Bedingungen angeben, die eine menschliche Siedlungsform erfüllen muss, um eine Stadt zu sein? Oder ist ein solches Unterfangen von Anfang an aussichtslos, ist die historische wie kulturelle Varianz des Phänomens Stadt so groß, dass es nicht möglich ist, einen begrifflichen Kern zu bestimmen, der sich in jeder Stadt, zu jeder Zeit und an jedem Ort manifestiert ${ }^{13}$ Neben dem Stadtbegriff müsste eine derart orientierte Philosophie der Stadt etwa auch Begriffe wie Zentrum, Metropole, Urbanität, Stadtgesellschaft und Land (als Gegensatz zur Stadt) betrachten.

Neben begrifflichen Fragen stellen sich innerhalb dieses Paradigmas wichtige epistemische Fragen, von denen zwei eng miteinander verknüpfte hier genannt werden sollen. Dies ist zum einen die Frage, welche Art von

12 Vgl. dazu Carlson (2014, bes. 2of.), der anschaulich darstellt, wie sich in den letzten Jahrzehnten aus der jüngeren environmental aesthetics die aesthetics of human environments / urban aesthetics schrittweise entwickelt hat.

13 Für ein ebenso aktuelles wie diskussionswürdiges Beispiel einer philosophischen Definition des Stadtbegriffs vgl. Varzi (2021), der die Stadt als einen sich in der Zeit entfaltenden Prozess bestimmt. 
Wissen im Hinblick auf Städte überhaupt möglich ist. Ist dieses Wissen lokal gebunden, das heißt, ist es Wissen über eine konkrete Stadt (ggf.: zu einem bestimmten Zeitpunkt) - oder lässt sich Wissen über „die Stadt“ im Allgemeinen durch Forschung produzieren, ist eine allgemeine Erkenntnis dessen möglich, was Städte ausmacht, was städtische Lebensformen kennzeichnet, was für urbane Strukturen prägend ist etc.? Anders formuliert: Gleicht Wissen über die Stadt eher dem historischen Wissen, wie es die Geschichtswissenschaften erarbeiten und wie es meist bestimmte Sachverhalte zu bestimmten Zeitpunkten an bestimmten Orten zum Inhalt hat - oder gleicht es einem soziostrukturellen Wissen, wie viele Sozialwissenschaften es zu erwerben suchen, indem durch lokal orientierte Studien Zusammenhänge erschlossen werden, die sich prinzipiell andernorts auch nachvollziehen und nachweisen lassen? Zum anderen stellt sich die Frage, welche Form der Stadttheorie eigentlich möglich ist. Lässt sich eine allgemeine, zum Beispiel soziologische Theorie der Stadt entwickeln, die auf zumindest hinreichend viele Städte Anwendung finden und von der die Stadtforschung entsprechend breit profitieren kann? Oder sind theoretische Annahmen eng an konkrete empirische Untersuchungen anzuschließen, ist also bestenfalls eine bedingte Abstraktion von Einzelfallergebnissen möglich, die zum Beispiel als Grundlage von Vergleichen herangezogen werden kann, deren Aussagekraft aber begrenzt bleibt? Fragen wie diesen sollte eine Philosophie der Stadt nachgehen, wenn sie sich primär als wissenschaftsphilosophische Reflexion disziplinärer und interdisziplinärer Stadtforschung versteht.

Stadtforschende aus verschiedenen Disziplinen betrachten ihr eigenes Forschungsfeld nicht selten auch als normatives Unterfangen: So wird behauptet, Stadtplanung und die ihr zuarbeitende Stadtforschung hätten progressiv zu sein, Prozesse der Emanzipation, der Inklusion und der Demokratisierung zu befördern. ${ }^{14}$ Mit Blick auf solche Positionsnahmen zeigt sich besonders deutlich eine mögliche dritte Auffassung der Aufgaben und Zielsetzungen einer Philosophie der Stadt: Sie kann als Normwissenschaft verstanden werden, die der Frage nachgeht, wie die für Stadtforschung relevanten normativen Begriffe, Regeln und Prinzipien zu interpretieren und zu bestimmen sind. An erster Stelle ist hier zweifelsohne der Gerechtigkeitsbegriff zu nennen, befassen sich doch viele Studien zur Stadt mit Fragen städ-

14 So ist etwa die Literatur zum emancipatory planning Legion; vgl. dazu die Hinweise im Text von Eckardt in diesem Schwerpunkt sowie als programmatisches Beispiel Albrechts (2003). 
tischer (Un-)Gerechtigkeit - sei es im Rahmen von Debatten zur Gentrifizierung, der Nutzung des öffentlichen Raumes, der Stadtentwicklung, der Smart Cities, der Möglichkeiten städtischer Partizipation oder der Integration. Natürlich hängen die Ergebnisse solcher Studien entscheidend von der Frage ab, welcher Gerechtigkeitsbegriff den jeweiligen Urteilen über Gerechtigkeit und Ungerechtigkeit zugrunde gelegt wird. Und wer sollte besser als Philosophierende dazu in der Lage sein, die Plausibilität von Gerechtigkeitskonzeptionen kritisch zu hinterfragen? Gleiches ließe sich auch im Hinblick auf Begriffe wie Inklusion, Nachhaltigkeit, Verantwortung oder Partizipation sagen: Eine Philosophie der Stadt als Normwissenschaft kann - ähnlich wie die Rechtswissenschaft - dazu beitragen, die in der Stadtforschung allgegenwärtigen normativen Urteile und Folgerungen zu präzisieren und, wo möglich, mit größerer Überzeugungskraft auszustatten.

Wie bereits gesagt: Diese drei Auffassungen schließen sich nicht gegenseitig aus; sie verdeutlichen vielmehr, dass die Philosophie sehr vieles und viel Verschiedenes zu Stadtdiskursen beitragen kann. Die Differenzierung deutet an, in welche Richtungen sich eine stärker an Konturen gewinnende Philosophie der Stadt weiterentwickeln kann. Hierzu will der vorliegende Schwerpunkt einen Beitrag leisten.

Die ersten beiden Texte des Schwerpunkts lassen sich der ersten und zweiten der oben skizzierten Auffassungen über die Aufgaben einer Philosophie der Stadt zuordnen. Verena Frick argumentiert in ihrem Beitrag „Braucht die Demokratie mehr städtische Autonomie? Zur politischen Philosophie der demokratischen Stadt“, dass die Stadt als eigenständiger politischer Handlungsraum zu verstehen sei, der eines spezifischen urbanen Demokratiebegriffs bedürfe. Marlon Barbehön und Michael Haus vertreten in „Jenseits von Souveränität und Territorialität: Überlegungen zu einer politischen Theorie der Stadt" die These, dass die Stadt im Rahmen der politischen Theorie nicht sinnvoll unter Rückgriff auf Kategorien staatsbezogener Souveränitätskonzeptionen zu begreifen, sondern als eigenständige Form räumlicher Vergesellschaftung zu denken ist, deren hervorstechende Merkmale Verdichtung und Heterogenisierung sind. Beide Texte schließen an etablierte Theorietraditionen an, wenn sie demokratische Leitbilder der Stadt analysieren (Frick) und stadtbezogene raumtheoretische Überlegungen (Barbehön/Haus) entwickeln. Zugleich geht es ihnen um eine konzeptionelle Grundlegung, die den Spezifika der Stadt und des Städtischen angemessen Rechnung trägt und die damit auch für die empirische, politikbezogene Stadtforschung theoretisch-begriffliche Grundlagen bereitstellt. 
In den drei folgenden Beiträgen des Schwerpunkts lässt sich sowohl die erste als auch die dritte Auffassung über den Aufgabenbereich einer Philosophie der Stadt wiederfinden. Im Rückgriff auf politikphilosophische Debatten untersuchen Frank Dietrich und Jonathan Seim in ihrem Text „Kommunale Online-Partizipation - Wer ist gefragt?“, wie der viele Stadtdiskurse prägende Begriff der Partizipation mit Blick auf das Fallbeispiel kommunaler digitaler Bürgerbeteiligung plausibel auszudeuten ist. Sie arbeiten heraus, dass Verfahren der Online-Beteiligung die Gefahr der Überinklusivität bergen, und zeigen die Notwendigkeit von normativen Kriterien zur Eingrenzung des städtischen Demos auf. Hier zeigt sich exemplarisch, wie politikphilosophische Theorien auf stadtpolitische Fragen bezogen werden und so zur besseren Bestimmung weitverbreiteter Normvorstellungen beitragen können.

Problemen städtischer Gerechtigkeit, wie sie oftmals unter dem Stichwort des „Rechts auf Stadt“ diskutiert werden, sind die Beiträge von Niklas Angebauer und Tobias Habermann sowie von Anke Strüver und Bernd Prien gewidmet. Angebauer und Habermann konstatieren in „Wem gehört die Stadt? Eigentum in der Debatte um bezahlbares Wohnen“, dass es sich bei der aktuellen „Wohnungskrise“ tatsächlich um eine „Bodenkrise“ handelt, die maßgeblich durch die eigentumspolitische Rahmung des Boden- und Immobilienmarktes beeinflusst ist. Sie argumentieren auf Basis dieser Diagnose für einen immanenztheoretischen Eigentumsbegriff, der keine absoluten, sondern qualifizierte Eigentumsrechte impliziert und dadurch eine Gemeinwohlbindung der Bodennutzung ermöglicht. Strüver und Prien greifen auf Nancy Frasers Gerechtigkeitstheorie zurück, um zu klären, wie ein „Recht auf digitale Stadt“ inhaltlich zu bestimmen ist. Im Mittelpunkt ihrer Überlegungen stehen Smart-City-Konzeptionen und der digitale Plattformurbanismus. Strüver und Prien verdeutlichen in ihrem Beitrag, wie eine etablierte philosophische Theorie zum besseren Verständnis wichtiger Phänomene zeitgenössischer urbaner Entwicklungen herangezogen werden und dazu beitragen kann, die zunächst unterbestimmte allgemeine Forderung nach Gerechtigkeit in spezifischen Kontexten theoriegeleitet zu konkretisieren. Anwendungsorientierte Studien sind für die Praktische Philosophie insgesamt von Nutzen, als sie es ermöglichen, den eigenen Theoriebestand am Beispiel kritisch auf Praktikabilität und Realitätsbezug hin zu überprüfen.

Der letzte Beitrag des Schwerpunkts, Frank Eckardts Text „Die Stadt als Assemblage: Neue Perspektiven für die Stadtplanung durch die Actor-Network-Theorie?", lässt sich als Beitrag zur zweiten der genannten Auf- 
fassungen über die Aufgaben einer Philosophie der Stadt verstehen. Eckardt geht am Beispiel von vier Fallstudien der Frage nach, inwiefern eine an der Planungspraxis orientierte Stadttheorie mit Gewinn auf Grundeinsichten der Akteur-Netzwerk-Theorie wie der Philosophie der Assemblage zurückgreifen kann. In diesem Rahmen wirft Eckardt auch die für die Philosophie der Stadt zentrale Frage auf, ob eine allgemeine Theorie der Stadt überhaupt möglich ist oder ob der Gegenstand Stadt nicht lediglich ein an Einzelbeispielen orientiertes „Theoretisieren“ über „die“ Stadt zulässt.

Die Beiträge zeigen die Vielfalt einer Philosophie der Stadt auf. Die Philosophie der Stadt wird davon profitieren, unterschiedliche philosophische Theorien und Methoden auf ihr stadtphilosophisches Potential hin zu befragen. Denn erst auf der Basis einer großen und inhaltlich diversen Zahl an stadtphilosophischen Erkundigungen wird das Feld mittelfristig an begrifflicher Kontur gewinnen und damit die interdisziplinäre Stadtforschung insgesamt stärker prägen können. Die Texte verdeutlichen, dass die Philosophie der Stadt den Austausch mit den anderen Disziplinen der empirischen Stadtforschung wie der Stadttheorie suchen muss. Sie zeigen zugleich, dass die Philosophie in einem entsprechend breiten Stadtdiskurs nicht nur nimmt, sondern anderen Disziplinen auch viel zu geben hat, zuallererst begriffliche Analysen und präzise und anwendungsbezogen ausgedeutete normative Leitvorstellungen.

\section{Literatur}

Albrechts, Louis. 2003. „Planning and Power: Towards an Emancipatory Planning Approach“. Politics and Space 21(6): 905-924. https://doi.org/10.1068/c29m.

Arendt, Hannah. 2002. Vita activa oder Vom tätigen Leben. München: Piper.

Benjamin, Walter. 1982. Das Passagen-Werk, 2 Bände, herausgegeben von Rolf Tiedemann. Frankfurt a.M.: Suhrkamp.

Brenner, Neil. 2011. Cities for People, Not for Profit: Critical Urban Theory and the Right to the City. London/New York: Routledge.

Calthorpe, Peter. 2013. Urbanism in the Age of Climate Change. Washington, D.C.: Island Press.

Carlson, Allen. 2014. „Ten Steps in the Development of Western Environmental Aesthetics“. In Environmental Aesthetics. Crossing Divides and Breaking Ground, herausgegeben von Martin Drenthen und Jozef Keulartz. New York: Fordham UP.

Dekker, Sabrina. 2020. Cities Leading Climate Action: Urban Policy and Planning. London/New York: Routledge.

Eckardt, Frank. 2004. Soziologie der Stadt. Bielefeld: transcript. 
Eckardt, Frank, Hrsg. 2017. Schlüsselwerke der Stadtforschung. Wiesbaden: Springer VS.

Epting, Shane, Hrsg. 2019. „Topical Issue: The Philosophical Dimensions of Urban Transportation“. Essays in Philosophy 20(2): 128-229.

Fields, Billy, und John L. Renne. 2021. Adaptation Urbanism and Resilient Communities. Transforming Streets to Address Climate Change. London/New York: Routledge.

Harvey, David. 1973. Social Justice and the City. Baltimore: John Hopkins University Press.

Harvey, David. 2019. Rebel Cities: From the Right to the City to the Urban Revolution. London: Verso.

Hasse, Jürgen, Hrsg. 2016. „Schwerpunkt: Philosophie der Stadt“. Forum Stadt 43(4): 323-408.

Horn, Philipp. 2020. Indigenous Rights to the City: Ethnicity and Urban Planning in Bolivia and Ecuador. London/New York: Routledge.

Jacobs, Jane. 1961. The Death and Life of Great American Cities. New York: Random House.

Jacobs, Keith, und Jeff Malpas, Hrsg. 2019. Philosophy and the City. Interdisciplinary and Transcultural Perspectives. London/New York: Rowman \& Littlefield.

Jaffe, Rivke, und Anouk De Koning. 2016. Introducing Urban Anthropology. London/New York: Routledge.

Jayne, Mark, und Kevin Ward, Hrsg. 2017. Urban Theory. New Critical Perspectives. London/New York: Routledge.

Lefebvre, Henri. 2016. Das Recht auf Stadt (Le droit à la ville. Paris: Anthropos, 1968). Hamburg: Edition Nautilus.

Lehtinen, Sanna, Hrsg. 2020a. „Topical Issue: Philosophy of the City“. Open Philosophy 3 (1).

Lehtinen, Sanna, Hrsg. 2020b. „Special Issue: Urban Aesthetics“. Contemporary Aesthetics Special Volume 8.

Löw, Martina. 2018. Vom Raum aus die Stadt denken. Grundlagen einer raumtheoretischen Stadtsoziologie. Bielefeld: transcript.

McLaren, Duncan, und Julian Agyeman. 2017. Sharing Cities: A Case for Truly Smart and Sustainable Cities. Cambridge, Mass./London: MIT Press.

Meagher, Sharon M., Hrsg. 2008. Philosophy and the City. Classic to Contemporary Writings. New York: State of New York UP.

Meagher, Sharon M., Samantha Noll und Joseph S. Biehl, Hrsg. 2019. The Routledge Handbook of Philosophy of the City. London/New York: Routledge.

Mitchell, Don. 2012. The Right to the City: Social Justice and the Fight for Public Space. New York/London: Guilford Press. 
Pardo, Italo, Hrsg. 2012. Anthropology in the City. Methodology and Theory. London/New York: Ashgate/Routledge.

Platon. 1981. Werke in acht Bänden. Bd. 5, bearb. v. Dietrich Kurz. Darmstadt: WBG.

Sassen, Saskia. 2001. The Global City: New York, London, Tokyo (urspr. 1991). Princeton: Princeton UP.

Siehr, Angelika. 2017. Das Recht am öffentlichen Raum: Theorie des öffentlichen Raumes und die räumliche Dimension von Freiheit. Tübingen: Mohr Siebeck.

Simon, Jules, Hrsg. 2021. „Special Issue: Introducing Philosophy of the City“. Topoi 40(2): 387-502.

Simmel, Georg. 2006. Die Großstädte und das Geistesleben (urspr. 1903). Frankfurt a.M.: Suhrkamp.

Varzi, Achille C. 2021. „What is a City?“. Topoi 40(2): 399-408.

Young, Iris Marion. 1990. Justice and the Politics of Difference. Princeton: Princeton University Press. 